\title{
The prevalence and characteristics of moderate- to high-risk regulated and unregulated substance use among patients admitted to four public hospitals in Tshwane, South Africa
}

\author{
A P Scheibe, MB ChB, MPH; N R Gloeck, MB ChB, DTM\&H; S Shelly, PG Dip Addictions Care; T S Marcus, BSocSci, MSc, PhD (Sociology); \\ J Hugo, MB ChB, MFamMed
}

Department of Family Medicine, School of Medicine, Faculty of Health Sciences, University of Pretoria, South Africa

Corresponding author: A Scheibe (andrew.scheibe@gmail.com)

\begin{abstract}
Background. Alcohol, tobacco and unregulated substance use contributes to the global burden of disease. Admission to hospital provides an opportunity to screen patients for substance use and offer interventions.

Objectives. To determine the prevalence and nature of substance use and treatment as well as interest in harm reduction among inpatients from four hospitals in the City of Tshwane, South Africa.

Methods. In a cross-sectional study, sociodemographic and substance use data were collected from 401 patients using the World Health Organization's Alcohol, Smoking and Substance Involvement Screening Test. Demographic characteristics were analysed using descriptive statistics. Bivariate and multivariate analyses of moderate- to high-risk tobacco and unregulated substance use in relation to demographic characteristics were also done.

Results. Most patients were South African (88\%) and black African (79\%), over half were female (57\%), and they were relatively young (median age 38 years). Most (82\%) lived in formal housing. Over half (56\%) had completed high school, and 33\% were formally employed. Bivariate analysis found substance use-related admission to be higher where scores for tobacco and unregulated substance use were moderate to high $(13 \%$ v. $0.3 \%, p<0.05)$. A notably higher $(p<0.1)$ proportion of participants with no/low tobacco and unregulated substance use had completed high school, were employed and were cohabiting/married compared with those with moderate to high scores. Across the hospitals, $32 \%$ (129/401) of the participants had moderate- to high-risk use of at least one substance: tobacco $(28 \%, 111 / 401)$, alcohol (10\%, $40 / 401)$, cannabis $(7 \%, 28 / 401)$, opioids $(2 \%, 9 / 401)$ and sedatives $(2 \%, 9 / 401)$. Of these 129 participants, $10 \%$ had accessed professional help, many $(67 \%, 78 / 129)$ wanted to learn more about harm reduction, and most $(84 \%, 108 / 129)$ said that they were willing to participate in a community-based harm reduction programme. Multivariate analysis found moderate- to high-risk tobacco and unregulated substance use to be positively associated with male sex (adjusted odds ratio (aOR) 7.9, 95\% confidence interval (CI) 2.9 - 21.5), age <38 years (aOR 3.3, 95\% CI 1.2 - 8.9), moderate- to high-risk alcohol use (aOR 3.1, 95\% CI $1.1-8.4 ; p=0.027$ ) and being admitted to Tshwane District Hospital (aOR 3.6, 95\% CI 1.1 - 12.2). It was negatively associated with employment (aOR 0.2, 95\% CI $0.1-0.6$ ).

Conclusions. Moderate- to high-risk substance use is an undetected, unattended comorbidity in the hospital setting in Tshwane, particularly among young, single, unemployed men. Clinicians should identify and respond to this need. Further research is required on the implementation of in-hospital substance use screening and treatment interventions.
\end{abstract}

S Afr Med J 2019;109(12):971-977. https://doi.org/10.7196/SAMJ.2019.v109i12.13870

Use of regulated (e.g. alcohol and nicotine) and unregulated (e.g. heroin, cannabis and cocaine) substances is common and a routine part of individual and collective social interaction. About $6 \%$ of the world's population use an unregulated substance, ${ }^{[1]}$ and a third of Africa's population report regular alcohol use. ${ }^{[2]}$ Whether these substances are intended to aid relaxation, increase energy and motivation or relieve boredom, ${ }^{[3-6]}$ certain patterns of use contribute to negative health and social outcomes and are linked to poor health, crime, family disruption, economic instability and conflict. ${ }^{[7]}$

Unregulated substances, usually bought off the street, hold additional dangers independent of the substance. Contamination with other substances and unknown concentration increase the risk of poisoning and overdose. ${ }^{[1]}$ Substances can be consumed through various routes, not limited to ingestion, smoking and injecting. The relative risks of overdose and the transmission of blood-borne infections are higher through injecting than other routes. Lack of sterile injecting equipment and subsequent sharing of needles increases the risk of HIV infection 22 -fold, ${ }^{[8]}$ and hepatitis $\mathrm{C}$ is at least 10 times more infectious per injecting event than HIV. ${ }^{[9]}$ This said, multi-criteria decisional analyses on the relative harms of regulated and unregulated substances in the $\mathrm{UK}^{[10]}$ and Europe ${ }^{[11]}$ suggest that alcohol creates the most harms to individuals and society, trumping all other substances, including heroin. In South Africa (SA), alcohol is the leading risk factor for disability-adjusted life-years and mortality, ${ }^{[10,12]}$ and there are global and event-level associations between alcohol consumption and poor adherence to antiretroviral therapy. ${ }^{[13,14]}$ Further, more than one-third of HIV-positive patients engage in heavy episodic drinking. ${ }^{[15]}$ In multi-criteria decision analysis, tobacco ranks between cocaine and amphetamines in terms of potential harms. ${ }^{[10]}$ The negative effects of smoking tobacco are well documented, and longstanding tobacco use diminishes overall health, increases health service utilisation and cost, and reduces individual functionality and productivity. ${ }^{[16]}$ The use of more than one substance is common, and has been reported by $40-56 \%$ of patients accessing specialised substance use disorder treatment centres in SA. ${ }^{[17]}$ Polysubstance use (the use of several substances 
within a specified period of time) increases potential health risks of individual substances and the burden on the health system. ${ }^{[18]}$

\section{Objectives}

Given limited systematic information but widespread tacit knowledge of alcohol and other substance use in the SA general population, ${ }^{[19]}$ there is a need to find out about their use among people who have been hospitalised to inform screening and interventions in the inpatient setting. This study describes the prevalence of regulated and unregulated substance use, substance use treatment seeking and interest in harm reduction among patients admitted to four hospitals in the City of Tshwane. The study also sought to characterise individuals at risk to inform screening and treatment interventions in the hospital setting. The study focused on inpatients for logistical reasons and interest around substance use and clinical care in the inpatient setting. It forms part of the Community Orientated Substance Use Programme (COSUP) being implemented by the University of Pretoria's Department of Family Medicine.

\section{Methods \\ Study setting}

The study was conducted in four hospitals. These hospitals form the Tshwane Steve Biko Academic Hospital Cluster that services five of the seven subdistricts in the Tshwane Metropolitan Municipality and is part of the University of Pretoria's academic platform. The hospitals predominantly serve the majority of the middle to low socioeconomic segments of the population. Steve Biko Academic Hospital is a tertiary hospital with 832 beds located in central Pretoria. Patients are referred from surrounding health facilities or admitted through the emergency/casualty unit. Kalafong Hospital is a tertiary hospital with 857 beds servicing the western areas of the city. Tshwane District Hospital is a 200-bed district hospital that drains the city's central business district and surrounds. Mamelodi Hospital, with $\sim 325$ beds, is a secondary hospital servicing Mamelodi township (an urban black African settlement created under the apartheid system) and surrounds.

\section{Study design}

This was a cross-sectional descriptive study.

\section{Study population and sampling}

The study population comprised patients aged $\geq 16$ years admitted to any one of the four hospitals. Patients aged $<16$ years, those in the intensive and high-care units of the hospitals, those who were too ill to answer the questions and those who declined to take part were excluded.

\section{Sample size and selection}

The sample size was calculated using $G^{*}$ Power, March 2014 version (University of Dusseldorf). The sample size was based on the assumption that the proportion of people admitted to hospital with moderate- to high-risk substance use was $10 \%$. A sample of 400 enabled this proportion to be estimated with a precision of $\pm 3 \%$ (95\% confidence interval (CI)). A total of 401 participants were recruited, with sample sizes proportional to total hospital inpatient capacity (425 patients were approached and 24 declined, giving a response rate of $94 \%)$. Systematic sampling was used to select participants. Study team members numbered beds in each ward, assigning each bed a number between 1 and 5 . Beds were numbered sequentially in a clockwise manner with the first bed being bed 1. In each ward, a random bed was selected as the starting point for bed selection using a random number generator. Thereafter, the occupant of every 5 th bed was included. When a bed was not occupied at the time of the interview, or the patient declined participation, the occupant of the bed immediately after and then immediately before the bed on the list was approached. Given that bed occupancy in wards varied, recruitment continued in each hospital until the subset sample size was reached.

\section{Data collection}

Data were collected using two instruments, both administered by a research assistant, done face to face and in sequence. A sociodemographic survey was used to collect data on age, biological sex, race, relationship, dwelling type, employment status and education level. The World Health Organization (WHO)'s Alcohol, Smoking and Substance Involvement Screening Test (ASSIST) ${ }^{[20]}$ was used to collect information on alcohol, tobacco and unregulated substance use.

ASSIST provides a calculation of the level of risk. Disaggregated by substance, risk levels are grouped into three categories, low, moderate and high, and attributed appropriate responses. People with low-risk scores were deemed not to warrant intervention. Those with moderateor high-risk scores related to any substance were deemed to require intervention. The research assistant conducted a brief intervention using the WHO 'feedback, responsibility, advice, menu of options, empathy and self-efficacy' or FRAMES motivational interviewing approach $^{[21]}$ and offered referral to additional substance use services, introducing COSUP. Interested participants were provided with an information leaflet on COSUP. For participants whose risk scored moderate to high, the sociodemographic questionnaire had additional questions on previous help-seeking behaviour and previous experiences in hospitals relating to substance use. They also received introductory information about harm reduction from those conducting the interview and were invited to attend a COSUP site on discharge for more information.

Data were collected over a period of 4 weeks on electronic devices using Qualtrics, November 2017 version (Qualtrics, USA), a University of Pretoria cloud-based web survey platform.

\section{Data analysis}

Categorical variables are presented as frequency tables and numerical variables as descriptive measures. Associations between moderate- to high-risk use of tobacco and an unregulated substance and demographic and substance use characteristics were assessed using bivariate and multivariate logistic regression analysis. For the regression analysis, several variables were converted into binary variables. Converted variables included age ( $<38$ years or $\geq 38$ years, the median age), race (black African or other), education (completed at least high school or not), dwelling type (formal housing or other) and livelihood (employed or unemployed). Kalafong Hospital was used as the reference hospital for interhospital comparison. Odds ratios (ORs) were used to measure associations in the bivariate analysis and adjusted ORs (aORs) for multivariate analysis. The multivariate regression analysis included sociodemographic characteristics and alcohol use. Data were analysed using Stata v11 (StataCorp, USA).

\section{Approval and consent}

The study was approved by the University of Pretoria's Faculty of Health Sciences Research Ethics Committee (ref. no. 369/2017) and the relevant hospitals. Respondents gave written informed consent before being interviewed. There was no reimbursement for participation. 


\section{Results}

In terms of sociodemographic characteristics and hospital entry pathways (Table 1), the median age of the respondents was 38 years (interquartile range (IQR) 28 - 50). Most were SA citizens (88\%) and black African (79\%), and lived in formal housing (82\%). Over half (57\%) were female, and over half (56\%) had completed high school.

\begin{tabular}{|c|c|}
\hline \multicolumn{2}{|l|}{ Hospital, $n(\%)$} \\
\hline Kalafong & $136(33.9)$ \\
\hline Mamelodi & $70(17.4)$ \\
\hline SBAH & $155(38.7)$ \\
\hline $\mathrm{TDH}$ & $40(10.0)$ \\
\hline \multicolumn{2}{|l|}{ Sex, $n(\%)$} \\
\hline Male & $173(43.1)$ \\
\hline Female & $228(56.9)$ \\
\hline \multicolumn{2}{|l|}{ Race, $n(\%)$} \\
\hline Black African & $317(79.0)$ \\
\hline Age (years), median (IQR) & $38(28-50)$ \\
\hline \multicolumn{2}{|l|}{ Nationality, $n(\%)$} \\
\hline SA citizen & $351(87.5)$ \\
\hline \multicolumn{2}{|l|}{ Education, $n(\%)$} \\
\hline Completed high school & $225(56.1)$ \\
\hline Employed, $n(\%)$ & $134(33.4)$ \\
\hline \multicolumn{2}{|l|}{ Relationship status, $n$ (\%) } \\
\hline Married or cohabiting & $139(34.7)$ \\
\hline No. of children, median (IQR) & $2(1-3)$ \\
\hline \multicolumn{2}{|l|}{ Dwelling, $n(\%)$} \\
\hline Formal housing & $328(81.8)$ \\
\hline $\begin{array}{l}\text { Average income (ZAR/month), } \\
\text { median (IQR) }\end{array}$ & $1400(0-4000)$ \\
\hline \multicolumn{2}{|l|}{ Entry path, $n(\%)$} \\
\hline Emergency & $161(40.1)$ \\
\hline Elective admission & $51(12.7)$ \\
\hline Referral from other facility & $189(47.1)$ \\
\hline $\begin{array}{l}\text { Admission related to substance use, } \\
n(\%)\end{array}$ & $5(1.2)$ \\
\hline
\end{tabular}

Thirty-five percent of participants were cohabiting or married, and $33 \%$ were formally employed. The median household income was ZAR1 400 per month (equivalent to USD100). Forty percent of participants were admitted for emergencies, and five people were admitted in relation to a consequence of their substance use.

Table 2 sets out the extent and kinds of moderate to high substancerelated risk by sex. One-third of the participants $(32 \%, 129 / 401)$ had moderate to high scores for at least one of the substances included in the screening. Significantly more males $(50 \%, 87 / 173)$ than females $(18 \%, 42 / 228)$ met the moderate- to high-risk criteria and required some kind of intervention for their substance use.

Moderate to high ASSIST scores related especially to tobacco products $(28 \%, 111 / 401)$, and risk due to tobacco use was notably higher among men than women (44\% v. $15 \%)$. Twelve participants (3\%) were assessed to be in need of additional treatment and were referred to appropriate services.

Alcohol was the second most common substance with moderate to high ASSIST scores $(10 \%, 40 / 401)$, with a higher prevalence among males than females (19\% v. $3 \%)$.

Seven per cent $(28 / 401)$ of the participants had moderate to high cannabis risk scores, with a higher prevalence among males than females ( $15 \%$ v. $1 \%)$.

In terms of opioids, 11 respondents (3\%) reported having ever used a non-regulated opioid (specifically heroin, known locally as nyaope, which is usually smoked with cannabis and may contain tobacco and/ or other bulking agents). Eight respondents said that they had used a non-regulated opioid during the previous 6 months, and 7 had injected it. One respondent had injected on a weekly basis during the 3 months prior to the study. Nine respondents (2\%), 8 of whom were male, had moderate to high ASSIST scores for opioids.

Moderate to high ASSIST scores were less frequent for other substances assessed as part of the ASSIST.

In terms of polysubstance use (Table 3), 32 respondents (8\%) had moderate to high ASSIST scores for tobacco and an unregulated substance, mostly cannabis $(n=24,6 \%)$ and opioids $(n=8,2 \%)$, followed by sedatives and cocaine. Eleven (3\%) had moderate to high scores for alcohol and cannabis, and $9(2 \%)$ had moderate to high scores for tobacco, alcohol and cannabis.

Among respondents with moderate to high substance use scores, two-fifths $(43 \%, 55 / 129)$ thought they needed professional assistance in view of their use of substances. Three in five $(60 \%, 78 / 129)$ expressed interest in learning about a programme to help them reduce the health risks of their substance use without abstinence as a

Table 2. Overview of participants with moderate to high World Health Organization ASSIST scores ${ }^{*}$ by hospital and sex in Tshwane, South Africa, $2017(N=401)$

\begin{tabular}{|c|c|c|c|c|c|c|c|c|c|c|}
\hline \multirow[b]{2}{*}{ Substance } & \multicolumn{2}{|c|}{ Total $(N=401), n(\%)$} & \multicolumn{2}{|c|}{ Mamelodi $(N=70), n(\%)$} & \multicolumn{2}{|c|}{ SBAH $(N=155), n(\%)$} & \multicolumn{2}{|c|}{ TDH $(N=40), n(\%)$} & \multicolumn{2}{|c|}{ Kalafong $(N=136), n(\%)$} \\
\hline & $\begin{array}{l}\text { Male } \\
(N=173)\end{array}$ & $\begin{array}{l}\text { Female } \\
(N=228)\end{array}$ & $\begin{array}{l}\text { Male } \\
(N=32)\end{array}$ & $\begin{array}{l}\text { Female } \\
(N=38)\end{array}$ & $\begin{array}{l}\text { Male } \\
(N=74)\end{array}$ & $\begin{array}{l}\text { Female } \\
(N=81)\end{array}$ & $\begin{array}{l}\text { Male } \\
(N=17)\end{array}$ & $\begin{array}{l}\text { Female } \\
(N=23)\end{array}$ & $\begin{array}{l}\text { Male } \\
(N=50)\end{array}$ & $\begin{array}{l}\text { Female } \\
(N=86)\end{array}$ \\
\hline $\begin{array}{l}\text { Any } \\
\text { substance }\end{array}$ & $87(50.3)$ & $42(18.4)$ & $21(65.6)$ & $5(13.2)$ & $27(36.5)$ & $13(16.0)$ & $11(64.7)$ & $6(26.1)$ & $28(56.0)$ & $18(20.9)$ \\
\hline Tobacco & $76(43.9)$ & $35(15.4)$ & $20(62.5)$ & $3(7.9)$ & $24(32.4)$ & $13(16.0)$ & $9(52.9)$ & $6(26.1)$ & $23(46.0)$ & $13(15.1)$ \\
\hline Alcohol & $33(19.1)$ & $7(3.1)$ & $8(25.0)$ & $2(5.3)$ & $9(12.2)$ & 0 & $4(23.6)$ & 0 & $12(24.0)$ & $5(5.8)$ \\
\hline Cannabis & $26(15.0)$ & $2(0.9)$ & $6(18.8)$ & 0 & $5(6.8)$ & 0 & $6(35.3)$ & $1(4.3)$ & $9(18.0)$ & $1(1.2)$ \\
\hline Opioids & $8(4.6)$ & $1(0.4)$ & 0 & 0 & $2(2.7)$ & 0 & $4(23.5)$ & $1(4.3)$ & $2(4.0)$ & 0 \\
\hline Sedatives & $3(1.7)$ & $6(2.6)$ & 0 & $1(2.6)$ & $3(4.1)$ & 0 & 0 & 0 & 0 & $5(6.8)$ \\
\hline Cocaine & $3(1.7)$ & $1(0.4)$ & $1(3.1)$ & 0 & $1(1.4)$ & 0 & $1(5.9)$ & $1(4.3)$ & 0 & 0 \\
\hline ATS & 0 & $2(0.9)$ & 0 & $1(2.6)$ & 0 & $1(1.2)$ & 0 & 0 & 0 & 0 \\
\hline
\end{tabular}

ASSIST = Alcohol, Smoking and Substance Involvement Screening Test; SBAH = Steve Biko Academic Hospital; TDH = Tshwane District Hospital; ATS = amphetamine-type stimulant ${ }^{*}$ Moderate substance use cut-off is an ASSIST score of $\geq 4$, except for alcohol, which has a cut-off of 11 . 
precondition. Most participants $(84 \%, 108 / 129)$ said that they would potentially be willing to participate in a community-based substance use programme that used a harm reduction approach.

Thirteen participants (11/87 male and 2/42 female) with moderate to high substance use risk scores had sought professional help on at least one occasion, albeit not during the 12 months prior to the study. Help-seeking behaviour was highest among the few using cocaine $(2 / 4)$ and opioids (3/9), and lowest among those with moderate- to high-risk use of alcohol or tobacco (3/40 and 11/111, respectively). Their main reasons for seeking professional help were personal $(n=3)$, social pressure $(n=3)$ and hospital admission $(n=2)$. The most common services they received were inpatient rehabilitation services $(n=5)$ and counselling $(n=4)$. Eight participants reported having absconded from a health facility due to their substance use, 2 of whom had high-risk opioid use scores. Detailed reasons for absconding were not collected.

The results of regression analysis are shown in Table 4. Bivariate analysis showed statistically significant $(p<0.05)$ associations between moderate- to high-risk use of tobacco and an unregulated substance and being male (OR 5.3, 95\% CI $2.3-12.6$; $p<0.001$ ), being $<38$ years of age (OR 2.9, 95\% CI $1.3-6.5 ; p=0.008$ ), having moderate- to highrisk alcohol use (OR 5.1, 95\% CI $2.2-11.8$; $p<0.001$ ) and being at Tshwane District Hospital compared with Kalafong Hospital (OR 2.8, 95\% CI 1.1 - 7.6; $p=0.039$ ).

Multivariate analysis showed that moderate to high scores for tobacco and an unregulated substance were positively associated with male sex (aOR 7.9, 95\% CI $2.9-21.5 ; p<0.001$ ), age $<38$ years
(aOR 3.3, 95\% CI 1.2 - 8.9; $p=0.017$ ), a moderate to high alcohol risk score (aOR 3.1, 95\% CI $1.1-8.4 ; p=0.027$ ) and admission to Tshwane District Hospital compared with Kalafong Hospital (aOR 3.6, 95\% CI 1.1 - 12.2; $p=0.038$ ). Formal employment (aOR 0.2, 95\% CI 0.1 $0.6 ; p=0.004$ ) lowered the risk for a moderate to high score for tobacco and an unregulated substance. Being married or cohabiting was negatively associated with a moderate to high score for tobacco and an unregulated substance, but the association was not statistically significant (aOR 0.3, 95\% CI $0.1-1.0$; $p=0.053$ ).

\section{Discussion}

A third of the 401 patients admitted to four large public hospitals in Tshwane during the study period had moderate to high ASSIST scores for at least one substance.

The predominance of males with moderate to high scores for tobacco, alcohol and opioids aligns with the international literature. ${ }^{[16,22]}$ In the SA setting, it may be that harmful use of substances is more common among men than among women. However, it is also likely that women who use substances harmfully are less visible and face a range of additional barriers to accessing services. Some additional barriers that women experience in seeking help for harmful substance use include less access to resources (including transport), limited family support, absence of childcare services, and fear that contact with the criminal justice system and social welfare services may jeopardise their custody of their children. ${ }^{[23-26]}$ As such, given social expectations of women in their roles as caregivers ${ }^{[27]}$ they are more likely to be affected by

Table 3. Summary of moderate/high unregulated substance use in relation to moderate- to high-risk tobacco and alcohol use according to World Health Organization ASSIST scores ${ }^{\star}$ by hospital and sex in Tshwane, South Africa, $2017(N=401)$

\begin{tabular}{llll}
\hline Unregulated substance & Tobacco, $\boldsymbol{n}(\%)$ & Alcohol, $\boldsymbol{n}(\%)$ & Tobacco and alcohol, $\boldsymbol{n}(\%)$ \\
\hline Any ${ }^{\dagger}$ & $32(8.0)$ & $12(3.0)$ & $10(2.5)$ \\
Cannabis & $24(6.0)$ & $11(2.7)$ & $9(2.2)$ \\
Opioids (heroin) & $8(2.0)$ & $1(0.2)$ & $1(0.2)$ \\
Sedatives & $4(1.0)$ & $1(0.2)$ & $1(0.2)$ \\
Cocaine & $4(1.0)$ & 0 & 0 \\
ATS & $2(0.5)$ & 0 & 0 \\
ASSIST = Alcohol, Smoking and Substance Involvement Screening Test; ATS = amphetamine-type stimulant. & \\
${ }^{*}$ Moderate substance use cut-off is an ASSIST score of $\geq 4$, except for alcohol, which has a cut-off of 11. \\
'This variable reflects participants who reported use of at least one unregulated substance (cannabis, opioids, sedatives, cocaine, ATS, hallucinogens, other non-specified unregulated substance) \\
as well as use of tobacco and/or alcohol.
\end{tabular}

Table 4. Bivariate and multivariate associations with moderate to high World Health Organization ASSIST scores ${ }^{\star}$ for tobacco and a moderate to high score for an unregulated substance in Tshwane, South Africa, 2017

\begin{tabular}{|c|c|c|c|c|c|c|}
\hline Variable & OR & $p$-value & $95 \% \mathrm{CI}$ & aOR & $p$-value & $95 \% \mathrm{CI}$ \\
\hline Male sex & 5.3 & $<0.001$ & $2.2-12.6$ & 7.9 & $<0.001$ & $2.9-21.5$ \\
\hline Age $<38$ years & 2.9 & 0.008 & $1.3-6.5$ & 3.3 & 0.017 & $1.2-9.0$ \\
\hline Black African & 1.2 & 0.750 & $0.5-2.9$ & 0.8 & 0.644 & $0.2-2.5$ \\
\hline Education (completed high school) & 0.5 & 0.070 & $0.2-1.1$ & 0.8 & 0.626 & $0.3-2.0$ \\
\hline Dwelling (formal housing) & 0.5 & 0.135 & $0.2-1.2$ & 1.1 & 0.918 & $0.4-2.8$ \\
\hline Employed & 0.4 & 0.074 & $0.2-1.1$ & 0.2 & 0.004 & $0.1-0.6$ \\
\hline Married or cohabiting & 0.4 & 0.055 & $0.2-1.0$ & 0.3 & 0.053 & $0.1-1.0$ \\
\hline \multicolumn{7}{|l|}{ Hospital } \\
\hline Kalafong & & & & - & - & - \\
\hline Mamelodi & 1.5 & 0.435 & $0.6-3.8$ & 1.7 & 0.910 & $0.6-4.9$ \\
\hline SBAH & 0.4 & 0.079 & $0.1-1.1$ & 0.4 & 0.136 & $0.1-1.4$ \\
\hline $\mathrm{TDH}$ & 2.8 & 0.039 & $1.1-7.6$ & 3.6 & 0.038 & $1.1-12.2$ \\
\hline Alcohol (moderate to high) & 5.1 & $<0.001$ & $2.2-11.8$ & 3.1 & 0.027 & $1.1-8.4$ \\
\hline
\end{tabular}


stigma and discrimination, exploitation by law enforcement and judgement from health workers. ${ }^{[28]}$

Similar to research findings elsewhere, the present study found an inverse relationship between moderate- to high-risk substance use and employment. ${ }^{[29]}$ Being married or in a long-term relationship has been described as protective in many studies, ${ }^{[29-31]}$ but was not statistically significant in this study. The association between younger age and increased moderate- to high-risk substance use has also been described elsewhere. ${ }^{[32,33]}$ A number of studies show that substance use disorders resolve, often without intervention, at a predictable and constant rate ${ }^{[34-36]}$ as people 'mature out ${ }^{[37]}$ of risky substance use. As people secure permanent employment, enter into long-term relationships and take on the responsibilities of parenthood, these changing roles may displace the use of unregulated substances. ${ }^{[38]}$ However, moderate- to high-risk use of alcohol and tobacco tends to follow a longer trajectory, which may in part be due to socially embedded acceptability as well as the slower trajectory of alcoholand tobacco-related harms. ${ }^{[39-41]}$

The above findings suggest that there is congruence between SA and international data, and this could point to shared experiences of substance use and outcomes.

As expected, the most commonly used substances - alcohol and tobacco - are also the substances that carry the highest risk for harmful use, even though they are regulated. ${ }^{[10]}$ We found that moderate- to high-risk tobacco use is associated with moderate- to high-risk use of alcohol and unregulated substances. In its own right and as an indicator for polysubstance use, the use of tobacco needs to be recognised as a significant risk factor among people admitted to hospital, and people with moderate- to high-risk smoking should be screened for the use of alcohol and unregulated substances.

Further, about one in 10 adult patients admitted to hospitals in Tshwane have moderate- or high-risk alcohol use patterns.

According to the ASSIST risk scores, almost half of the male participants in the present study and a sixth of females required some form of intervention for alcohol or tobacco use. Because of the strong association between harmful alcohol and tobacco use, and the increased health risk of their combination, there is a need for integrated brief interventions in healthcare settings, including hospitals. ${ }^{[42]}$ Our findings point to the often-missed opportunity in healthcare settings to engage with issues of harmful substance use that have a direct bearing on patient treatment and health outcomes. The introduction of substance use screening and brief interventions as a standard of care could significantly reduce the negative impacts of these substances, and the associated costs. ${ }^{[43]}$

Cannabis was found to be the most common non-regulated substance used. While the health consequences of cannabis use are potentially less serious than those for the other non-regulated substances, there are social risks associated with its use, particularly the risk of exposure to the criminal justice system. Notwithstanding the 18 September 2018 Constitutional Court ruling that effectively decriminalised private use of cannabis and its production for private consumption (however, although the use of cannabis in a private setting is widely described as 'legal', this is not correct - cannabis is still considered a 'scheduled substance' in terms of the Drugs and Drug Trafficking Act No. 140 of 1992), there is a lot of discretion left in the hands of the police (South African Police Services Directive No. 1/1/4/1, September 2018) and quotas for arrest targets must still be met (senior South African Police Service members at Safer Western Cape dialogues, 13 and 14 November 2018, personal communication).

There were 11 patients with moderate- to high-risk heroin use (unregulated opioids) in the inpatient setting. It is widely reported that dependence on unregulated opioids places the individual at increased risk of morbidity and mortality, including from overdose. People who are dependent on opioids, particularly those who inject them, are at high risk of viral hepatitis, HIV and other blood-borne infections, a situation that may be compounded, if not driven, by the absence of sterile injecting equipment. ${ }^{[1]}$

Although in this study proportionately few respondents (3\%) were found to have moderate- to high-risk use of heroin, over the past decade there has been an upward trend in heroin use among patients admitted to Gauteng substance use disorder treatment centres that report admissions data to the South African Community Epidemiology Network on Drug Use. ${ }^{[17]}$ Of 3414 individuals admitted between June and December 2017, 14\% said that they used heroin as their primary drug, and it was the third most common substance used. ${ }^{[17]}$ The numbers in the present study are therefore likely to be an under-representation of heroin-dependent people needing hospital admission. ${ }^{[4]}$ Moreover, poor understanding of dependence and lack of provision of opioid agonists to address the clinical effect of opioid withdrawal, as well as limited professional understanding of pain management in these patients, may also contribute to the low numbers of people who use heroin being treated in these hospitals. Heroin-dependent patients often require higher doses of medications, ${ }^{[45]}$ including short-acting opioids, to deal with increased pain sensitivity ${ }^{[46]}$ There are also fears that prescribing opioids may promote development of an opioid use disorder, although it has been shown that appropriate prescribing seldom leads to substance use disorders. ${ }^{[47]}$

The findings in the present study that levels and type of substance use involvement varied between hospitals may be idiosyncratic. They may also suggest local specificity, reflecting different substance use dynamics as well as the particularities of clinical services in the inner city, suburbs and townships.

\section{Study limitations}

The results of this study may underestimate the prevalence of substance use among patients admitted to hospitals owing to sensitivities around harmful substance use, particularly where unregulated substances are involved. To some extent these fears may have been countered by staff, who were trained to conduct interviews in a neutral and non-judgemental manner and to reassure participants of the steps taken to protect their information. However, these efforts would not impact on the number of people who leave hospital while waiting to be attended to or during their admissions or emergency unit consultations. ${ }^{[48]}$ The study tools did not assess participants' understanding of harm reduction as a concept. As a result, responses related to their willingness to use harm reduction services may not have been measured accurately.

\section{Conclusions and recommendations}

The study highlights that a significant proportion of hospital patients use regulated and unregulated substances, often in a way that puts them at moderate to high risk. We identified a relationship between moderate- to high-risk use of tobacco and moderate- to high-risk use of unregulated substances. The causal relationship between potentially high-risk tobacco use and unregulated substance use cannot be assessed in a cross-sectional survey, but the study has identified substance use as an important area of healthcare in hospitals, particularly among young men facing socioeconomic hardship.

Additional assessment of the feasibility of using the WHO ASSIST, particularly the brief intervention linked to screening, is needed to further inform its role in hospital settings. 
There is a need for patients who are dependent on opioids or other substances to receive adjunctive interventions for the duration of their in-hospital treatment. As in other settings, and in line with international guidelines, opioid substitution therapy (OST) in hospitals should use adequate doses. On leaving hospital, patients should be offered the best available primary healthcare, including access to OST. For facilities in the City of Tshwane, for example, OST is increasingly available through COSUP. ${ }^{[49]}$

As per the Batho Pele principles (constitutionally based principles outlining the way in which public services should be provided: they are consultation, service standards, access, courtesy, information, openness and transparency, redress and value for money), all patients should receive non-judgemental services. Hospital staff should be adequately trained around the risks of regulated and unregulated substances and on brief interventions and referral. An assessment of hospital staff knowledge, attitudes and practices around substance use would be an important first step towards in-service and undergraduate health worker training.

From the perspective of patients, approaches that reduce the potential harms of substance use without necessarily requiring the individual to abstain are likely to elicit better responses to treatment, as will self-management, and should be evaluated further. Additional research to assess levels of substance use among hospital patients in emergency, casualty, maternity and outpatient units is also needed.

\section{Declaration. None.}

Acknowledgements. We thank Prof. Brendan Girdler-Brown for statistical guidance and input to the research protocol, Matome Mohale, Ofentse Mekgwe, Abiel Biza, Game Makhubele, Nonkululeko Khumalo, Thabo Papo, Oratiloe Moiloa, Zinhle Mazibuko and Nomthandazo Maphanga, medical students who assisted with data capturing, Abiel Biza, Zinhle Mazibuko and Nomthandazo Maphanga for assistance with data entry and quality checks, Dr Gerhard Botha and the Daspoort polyclinic team for piloting and training, and Lesego Sehume for administration support. Author contributions. APS co-developed the protocol, supported implementation, conducted the data analysis, drafted the initial version of the manuscript and co-ordinated and consolidated co-author inputs. NRG co-developed the protocol, led implementation, drafted the 'Background' and 'Methods' sections and provided inputs into the development of the manuscript. SS provided inputs into the protocol and into the development of the manuscript. TSM provided inputs into the protocol and into the development of the manuscript. JH provided inputs into the protocol and into the development of the manuscript.

Funding. The research was funded as part of COSUP, funded by the City of Tshwane and implemented by the University of Pretoria (UP) Department of Family Medicine and the UP Community Oriented Primary Care Research Unit.

Conflicts of interest. None.

1. United Nations Office on Drugs and Crime (UNODC). World Drug Report 2018. https://www.unode. org/wdr2018/ (accessed 26 September 2018).

2. World Health Organization. Global status report on alcohol and health 2018. https://www.who.int/ substance_abuse/publications/global_alcohol_report/en/(accessed 9 December 2018).

3. Boys A, Marsden J, Strang J. Understanding reasons for drug use amongst young people: A functional perspective. Health Educ Res 2018;16(4):457-469. https://doi.org/10.1093/her/16.4.457

4. Johnston LD, O'Malley PM. Why do the nation's students use drugs and alcohol? Selfreported reasons from nine national surveys. J Drug Issues 1986;16(1):29-66. https://doi org/10.1177/002204268601600103

5. Siegel RK. Intoxication: The Universal Drive for Mind Altering Substances. Simon \& Schuster, 2005. https://www.simonandschuster.com/books/Intoxication/Ronald-K-Siegel/9781594770692 (accessed 9 December 2018).

6. Morgan CJA, Noronha LA, Muetzelfeldt M, et al. Harms and benefits associated with psychoactive drugs: Findings of an international survey of active drug users. J Psychopharmacol 2013;27(6):497506. https://doi.org/10.1177/0269881113477744
Ramlagan S, Peltzer K, Matseke G. Epidemiology of drug abuse treatment in South Africa. S Afr J Psychiatry 2010;16(2):40-49. https://doi.org/10.4102/sajpsychiatry.v16i2.172

8. Joint United Nations Programme on HIV and AIDS (UNAIDS). Miles to go: Closing gaps, breaking barriers, righting injustices. 2018. http://www.unaids.org/en/resources/documents/2018/global-aidsupdate (accessed 10 October 2018).

9. Page K, Morris MD, Hahn JA, et al. Injection drug use and hepatitis $\mathrm{C}$ virus infection in young adult injectors: Using evidence to inform comprehensive prevention. Clin Infect Dis 2013;57(Suppl 2):S32-S38. https://doi.org/10.1093/cid/cit300

10. Nutt DJ, King LA, Phillips LD. Drug harms in the UK: A multicriteria decision analysis. Lancet 2010;376(9752):1558-1565. https://doi.org/10.1016/s0140-6736(10)61462-6

1. Van Amsterdam J, Nutt D, Phillips L, et al. European rating of drug harms. J Psychopharmacol 2015;29(6):655-660. https://doi.org/10.1177/0269881115581980

12. Segura L, Anderson P, Gual A. Optimizing the delivery of interventions for harmful alcohol use in primary healthcare. Curr Opin Psychiatry 2018;31(4):324-332. https://doi.org/10.1097/ yco.0000000000000435

13. Hendershot CS, Stoner SA, Pantalone DW, et al. Alcohol use and antiretroviral adherence: Review and meta-analysis. J Acquir Immune Defic Syndr 2009;52(2):180-202. https://doi.org/10.1097/ QAI.0b013e3181b18b6e

14. Kip E, Ehlers VJ, van der Wal DM. Patients' adherence to anti-retroviral therapy in Botswana. J Nurs Scholarsh 2009;41(2):149-157. https://doi.org/10.1111/j.1547-5069.2009.01266.x

15. Subramaniam P, McGlade E, Yurgelun-Todd D. Comorbid cannabis and tobacco use in adolescents and adults. Curr Addict Rep 2016;3(2):182-188. https://doi.org/10.1007/s40429-016-0101-3

16. Centers for Disease Control and Prevention. Health effects of cigarette smoking. 17 January 2018 (updated 20 May 2017). https://www.cdc.gov/tobacco/data_statistics/fact_sheets/health_effects/ effects_cig_smoking/index.htm (accessed 4 June 2018).

17. Dada S, Burnhams N, Erasmus J, et al. Alcohol and other drug use trends (South Africa): July to December 2017 (phase 43). South African Community Epidemiology Network on Drug Use (SACENDU), 2017. http://www.samrc.ac.za/sites/default/files/attachments/2018-07-26/ SACENDUupdateJun2018.pdf (accessed 12 September 2018)

18. Connor JP, Gullo MJ, White A, et al. Polysubstance use: Diagnostic challenges, patterns of use and health. Curr Opin Psychiatry 2014;27(4):269-275. https://doi.org/10.1097/YCO.0000000000000069
hen

19. Dada S, Harker Burnhams N, Erasmus J, et al. Update January 2018 alcohol and other drug use trends: January to June 2017 (phase 42). South African Community Epidemiology Network on Drug Use (SACENDU), 2018. http://www.mrc.ac.za/sites/default/files/attachments/2018-03-02/ SACENDUupdateJan2018.pdf (accessed 10 September 2018)

20. World Health Organization. The ASSIST project - Alcohol, Smoking and Substance Involvement Screening Test. 2010. https://www.who.int/substance_abuse/activities/assist/en/ (accessed 6 June 2018).

21. Rollnick S, Miller WR. What is motivational interviewing? Behav Cogn Psychother 1995;23(4):325334. https://doi.org/10.1017/S135246580001643X

22. Van Etten ML, Anthony JC. Male-female differences in transitions from first drug opportunity to first use: Searching for subgroup variation by age, race, region, and urban status. J Womens Health Gend Based Med 2004;10(8):797-804. https://doi.org/10.1089/15246090152636550

23. Moloko-Phiri SS, Mogale RS, Hugo J. 'A shelter is not a home': Voices of homeless women in the city of 3. Moloko-Phiri SS, Mogale RS, Hugo J. 'A shelter is not a home': Voices of homeless women in the
Tshwane. Dev South Afr 2017;34(4):439-449. https://doi.org/10.1080/0376835X.2017.1318048

24. Copeland J. A qualitative study of barriers to formal treatment among women who self-managed change in addictive behaviours. J Subst Abuse Treat 1997;14(2):183-190. https://doi.org/doi. org/10.1016/S0740-5472(96)00108-0

25. Neale J, Nettleton S, Pickering L. Gender sameness and difference in recovery from heroin dependence: A qualitative exploration. Int J Drug Policy 2014;25(1):3-12. https://doi.org/10.1016/j. drugpo.2013.08.002

26. Myers B, Louw J, Pasche S. Gender differences in barriers to alcohol and other drug treatment in Cape Town, South Africa. Afr J Psychiatry 2011;14(2). https://doi.org/10.4314/ajpsy.v14i2.7

27. United Nations Task Force on Transnational Organized Crime and Drug Trafficking as Threats to Security and Stability. A gender perspective on the impact of drug use, the drug trade, and drug control regimes. UN Women Policy Brief. July 2014. https://www.unodc.org/documents/ungass2016/ Contributions/UN/Gender_and_Drugs___UN_Women_Policy_Brief.pdf (accessed 6 June 2018).

28. Azim T, Bontel I, Strathdee SA. Women, drugs and HIV. Int J Drug Policy 2015;26(Suppl 1):S16-S21. https://doi.org/10.1016/j.drugpo.2014.09.003

29. Merline AC, O’Malley PM, Schulenberg JE, et al. Substance use among adults 35 years of age: Prevalence, adulthood predictors, and impact of adolescent substance use. Am J Public Health 2004;94(1):96-102. https://doi.org/10.2105/ajph.94.1.96

30. Robins LN, Reigers DA, eds. Psychiatric Disorders in America - the Epidemiologic Catchment Area Study. New York: The Free Press, Division of MacMillan, 1991.

31. Kandel D, Simcha-Fagan O, Davies M. Risk factors for delinquency and illicit drug use from adolescence to young adulthood. J Drug Issues 1986;16(1):67-90. https://doi.org/10.1177/002204268601600104

32. Kandel DB, Logan JA. Patterns of drug use from adolescence to young adulthood: I. Periods of risk for initiation, continued use, and discontinuation. Am J Public Health 1984;74(7):660-666. https://doi. org/10.2105/AJPH.74.7.660

33. Kandel DB, Raveis VH. Cessation of illicit drug use in young adulthood. Arch Gen Psychiatry 1989;46(2):109-116. https://doi.org/10.1001/archpsyc.1989.01810020011003

34. Heyman GM. Quitting drugs: Quantitative and qualitative features. Annu Rev Clin Psychol 2013:9:2959. https://doi.org/10.1146/annurev-clinpsy-032511-143041

35. Lopez-Quintero C, Hasin DS, de los Cobos JP, et al. Probability and predictors of remission from life-time nicotine, alcohol, cannabis or cocaine dependence: Results from the national epidemiologic survey on alcohol and related conditions. Addiction 2011;106(3):657-669. https://doi.org/10.1111/ j.1360-0443.2010.03194.x

36. White WL. Recovery/remission from Substance Use Disorders: An Analysis of Reported Outcomes in 415 Scientific Reports, 1868 - 2011. Chicago: Philadelphia Department of Behavioural Health and Intellectual Disability Services, 2012

37. Winick C. Maturing out of narcotic addiction. United Nations Office on Drugs and Crime (UNODC), 1962. https://www.unodc.org/unodc/en/data-and-analysis/bulletin/bulletin_1962-01-01_1_page002. html (accessed 9 December 2018).

38. Staff J, Schulenberg JE, Maslowsky J, et al. Substance use changes and social role transitions: Proximal Staff J, Schulenberg JE, Maslowsky J, et al. Substance use changes and social role transitions: Proximal
developmental effects on ongoing trajectories from late adolescence through early adulthood. Dev Psychopathol 2010;22(4):917-932. https:///doi.org/10.1017/s0954579410000544

39. Blomqvist J. Recovery with and without treatment: A comparison of resolutions of alcohol and drug problems. Addict Res Theory 2002;10(2):119-158. https://doi.org/10.1080/16066350290017248

40. Grant JD, Vergés A, Jackson KM, et al. Age and ethnic differences in the onset, persistence and recurrence of alcohol use disorder. Addiction 2012;107(4):756-765. https://doi.org/10.1111/j.13600443.2011.03721.x

41. Vergés A, Haeny AM, Jackson KM, et al. Refining the notion of maturing out: Results from the national epidemiologic survey on alcohol and related conditions. Am J Public Health 2013;103(12):e67-e73. https://doi.org/10.2105/AJPH.2013.301358

42. Pengpid S, Peltzer K, Puckpinyo A, et al. Screening and concurrent brief intervention of conjoint hazardous or harmful alcohol and tobacco use in hospital out-patients in Thailand: A randomized controlled trial. Subst Abuse Treat Prev Policy 2015;10(1):22. http:://doi.org/10.1186/s13011-015-0018-1 
43. Solberg LI, Maciosek MV, Edwards NM. Primary care intervention to reduce alcohol misuse ranking its health impact and cost effectiveness. Am J Prev Med 2008;34(2):143-152. https://doi.org/10.1016/j. amepre.2007.09.035

44. Meel R, Essop MR. Striking increase in the incidence of infective endocarditis associated with recreational drug abuse in urban South Africa. S Afr Med J 2018;108(7):585-589. https://doi. org/10.7196/SAMJ.2018.v108i7.13007

45. Compton P, Canamar CP, Hillhouse M, Ling W. Hyperalgesia in heroin dependent patients and the effects of opioid substitution therapy. J Pain 2012;13(4):401-409. https://doi.org/10.1016/j. jpain.2012.01.001

46. Eyler ECH. Chronic and acute pain and pain management for patients in methadone maintenance treatment. Am J Addict 2013;22(1):75-83. https://doi.org/10.1111/1.1521-0391.2013.00308.x
47. Noble M, Treadwell JR, Tregear SJ, et al. Long-term opioid management for chronic non cancer pain. Cochrane Database Syst Rev 2010, Issue 1. Art. No.: CD006605. https://doi.org/10.1002/14651858 CD006605.pub2

48. Ti L, Ti L. Leaving the hospital against medical advice among people who use illicit drugs: A systematic review. Am J Public Health 2015;105(12):2587-2587. https://doi.org/10.2105/ajph.2015.302885a

49. Research Matters. Drug intervention that works and makes you feel human again. University of Pesearch Matters. Drug intervention that works and makes you feel human again. University of
Pretoria, 2018. http://www.researchmatters.up.ac.za/researcher-projects/view/129 (accessed 12 December 2018).

Accepted 14 June 2019 